\title{
Initial Operation and Shakedown of the Nuclear Thermal Rocket Element Environmental Simulator (NTREES)
}

\author{
William J. Emrich, $\mathrm{Jr}^{1}$ \\ NASA Marshall Space Flight Center, Huntsville, Alabama, 35812
}

\begin{abstract}
To support the on-going nuclear thermal propulsion effort, a state-of-the-art non nuclear experimental test setup has been constructed to evaluate the performance characteristics of candidate fuel element materials and geometries in representative environments. The facility to perform this testing is referred to as the Nuclear Thermal Rocket Element Environment Simulator (NTREES). This device can simulate the environmental conditions (minus the radiation) to which nuclear rocket fuel components will be subjected during reactor operation. Prototypical fuel elements mounted in the simulator are inductively heated in such a manner so as to accurately reproduce the temperatures and heat fluxes which would normally occur as a result of nuclear fission in addition to being exposed to flowing hydrogen. Recent upgrades to NTREES now allow power levels 24 times greater than those achievable in the previous facility configuration. This higher power operation will allow near prototypical power densities and flows to finally be achieved in most prototypical fuel elements.
\end{abstract}

\section{Introduction}

$\mathrm{T}$ The NTREES facility is designed to perform realistic non-nuclear testing of nuclear thermal rocket (NTR) fuel elements and fuel materials and is licensed by the Nuclear Regulatory Commission to handle fuel elements containing depleted uranium. Although the NTREES facility cannot mimic the neutron and gamma environment of an operating NTR, it can simulate the thermal hydraulic environment within an NTR fuel element to provide critical information on material performance and compatibility. Over the past year the Nuclear Thermal Rocket Element Environmental Simulator (NTREES) has been undergoing a significant upgrade. The modifications to NTREES which have just been completed expand the power capabilities of the facility significantly. In particular, the previous $50 \mathrm{~kW}$ induction power supply has been replaced with a 1.2 MW unit allowing more prototypical fuel element temperatures to be reached ${ }^{1}$.

The new 1.2 MW induction heater consists of three physical units consisting of a transformer, rectifier, and inverter. This multiunit arrangement facilitated increasing the flexibility of the induction heater by more easily allowing variable frequency operation. Frequency ranges between 20 and $60 \mathrm{kHz}$ can accommodated in the new induction heater allowing more representative power distributions to be generated within the test elements. The water cooling system was also upgraded to so as to be capable of removing $100 \%$ of the heat generated during testing. This new setup required that the NTREES vessel be raised onto a platform along with most of its associated gas and vent lines. In this arrangement, the induction heater and water systems are now located underneath the platform.

\section{Facility Description}

In this new configuration, the 1.2 MW NTREES induction heater will be capable of testing fuel elements and fuel materials in flowing hydrogen at pressures up to 1000 psi at temperatures up to and beyond $3000 \mathrm{~K}$ and at nearprototypic reactor channel power densities. NTREES is also capable of testing potential fuel elements with a variety of propellants, including hydrogen with additives to inhibit corrosion of certain potential NTR fuel forms.

Additional diagnostic upgrades included in the present NTREES set up include the addition of a gamma ray spectrometer located near the vent filter to detect uranium fuel particles exiting the fuel element in the propellant exhaust stream to provide additional information any material loss occurring during testing. Other aspects of the upgrade included reworking NTREES to reduce the operational complexity of the system despite the increased

\footnotetext{
${ }^{1}$ Senior Engineer, Propulsion System Department, Mail Stop ER24, AIAA Member.
} 
complexity of the induction heater. The NTREES facility is also now equipped with some additional hardware for instrumentation, control, and data acquisition. The hardware is distributed around the test chamber and support equipment, but integrated and controlled from one piece of software running on one computer located in the control room. This new suite of sensors includes:

- Pressure sensors for $\mathrm{GH}_{2}$ and $\mathrm{GN}_{2}$

- Temperature sensors for $\mathrm{GH}_{2}$ and $\mathrm{GN}_{2}$

- Flow sensors for $\mathrm{GH}_{2}$ and $\mathrm{GN}_{2}$

- Thermocouples for general temperature measurements

- Hydrogen detection for the test chamber and room 101

- Pyrometers for temperature measurement of test pieces

- Mass spectrometer to measure gas composition

- Gamma ray spectrometer to detect uranium loss

In addition to the new sensors, software control program has been almost completely rewritten to allow more data to be displayed and to permit a more intuitive user interface which allows NTREES to be operated in a more user friendly manner.

Recently, NTREES underwent an Operational Readiness Review (ORI) and the facility was declared safe to begin operation. As part of the review process, the facility underwent a complete certification process for the pressure system along with a Safety Assessment (SA) and a review of the Standard Operating Procedures (SOPs). A considerable amount of the the changes to the SOP were to make sure that the potential for contamination of the facility and surrounding area was reduced or eliminated. Figure 1 illustrates the current configuration of the NTREES facility.

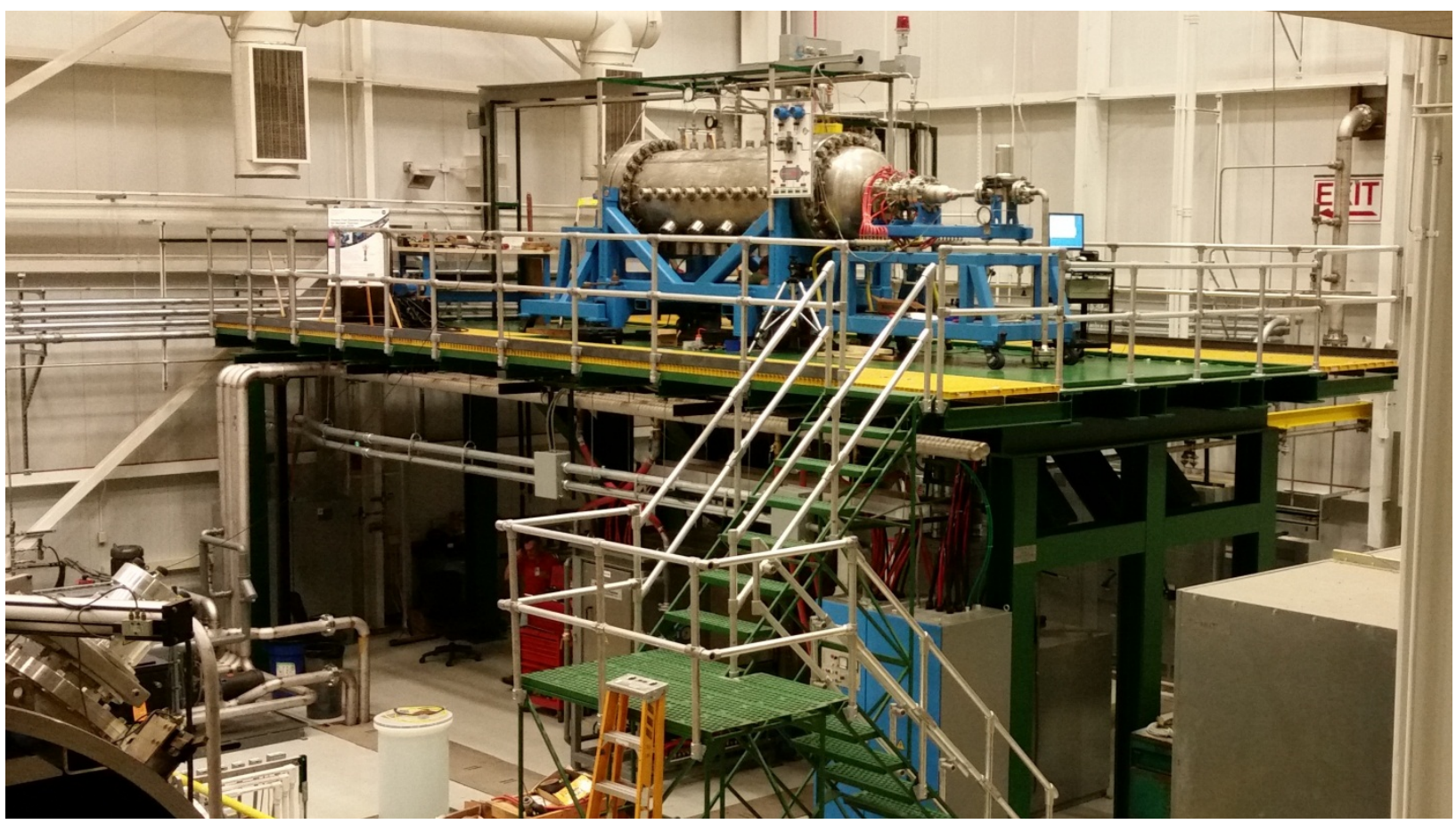

Figure 1 Nuclear Thermal Rocket Element Environmental Simulator (NTREES)

\section{Initial Operation}

Following the Operational Readiness Review, initial shake down operation of NTREES was begun. On the first test of the induction heater system, a short circuit occurred in the metal braided induction coil coolant lines. These 
lines supply water to the coil to prevent overheating of the coil when the fuel element is operating at the high temperatures characteristic of nuclear rocket operation. The short occurred due to movement of the coolant lines when they were pressurized for operation. Fortunately, only the coolant lines were damaged and the rest of the facility escaped unscathed. The new coolant lines were covered with insulation wrap and restrained to prevent excessive movement. Following these measures, NTREES was again powered up and no shorts occurred. Figure 2 illustrates the the NTREES induction coil and Figure 3 illustrates the severe damage to the coolant lines when only approximately $40 \mathrm{~kW}$ of power was applied to the induction coil!

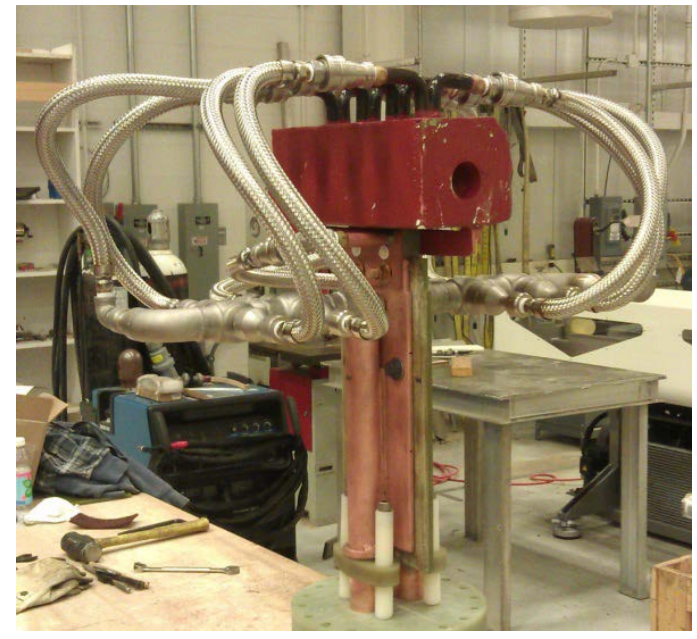

Figure 2 Induction Coil Assembly

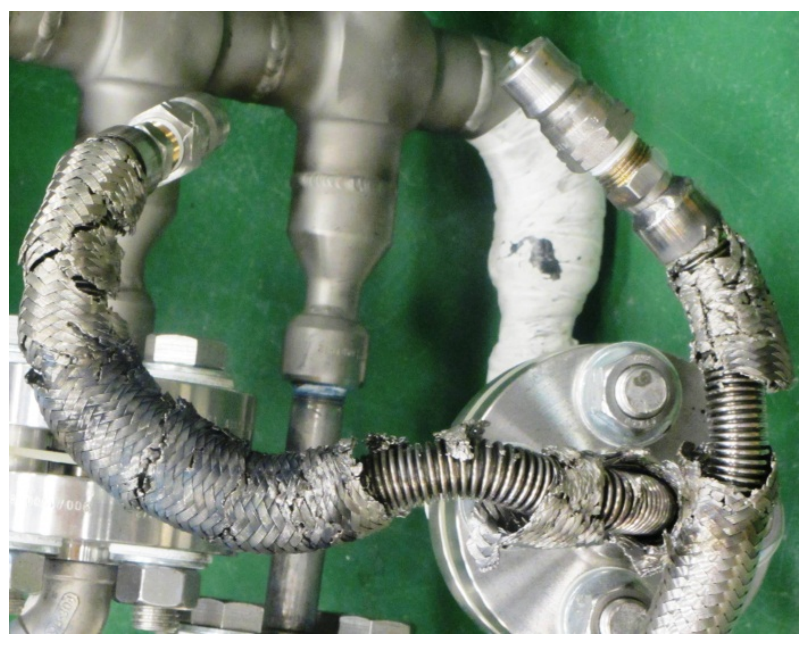

Figure 3 Damaged Induction Coil Hoses

As the power was increased in NTREES, an infrared camera was set up to search for hot spots in the setup. One area of special concern was the the feedthru assembly where the power from the induction heater is fed through the NTREES chamber to the coil. Care had been taken to provide cooling to the bussbar assembly leading to the feedthru and to the power leads which connected to the induction coil so there was reasonable confidence that there would be no heating problems. As it happened, we were wrong in our assumption that there would be no heating problems and the problem appeared in a most unexpected place. It turned out that the steel bolts connecting the feedthru flange to the chamber were coupling with the induction field and heating up. Since the flange is fabricated out of G10, which is a plastic insulating material, it is capable of maintaining its strength only at fairly low temperatures $\left(<250{ }^{\circ} \mathrm{C}\right)$. Hot bolts in the plastic flange were definitely a problem.

Since cooling the bolts was out of the question, a way had to be found to shield the bolts from the stray induction fields. It was decided to imbed the bolt heads in a new flange collar made of copper and to shield the bolt bodies themselves with copper sleeves. These modifications had the desired effect and reduced the bolt temperature to acceptable levels. Figure 4 shows the heating experienced by the bolts before the modification and Figure 5 shows the temperature reduction after the modifications were completed.
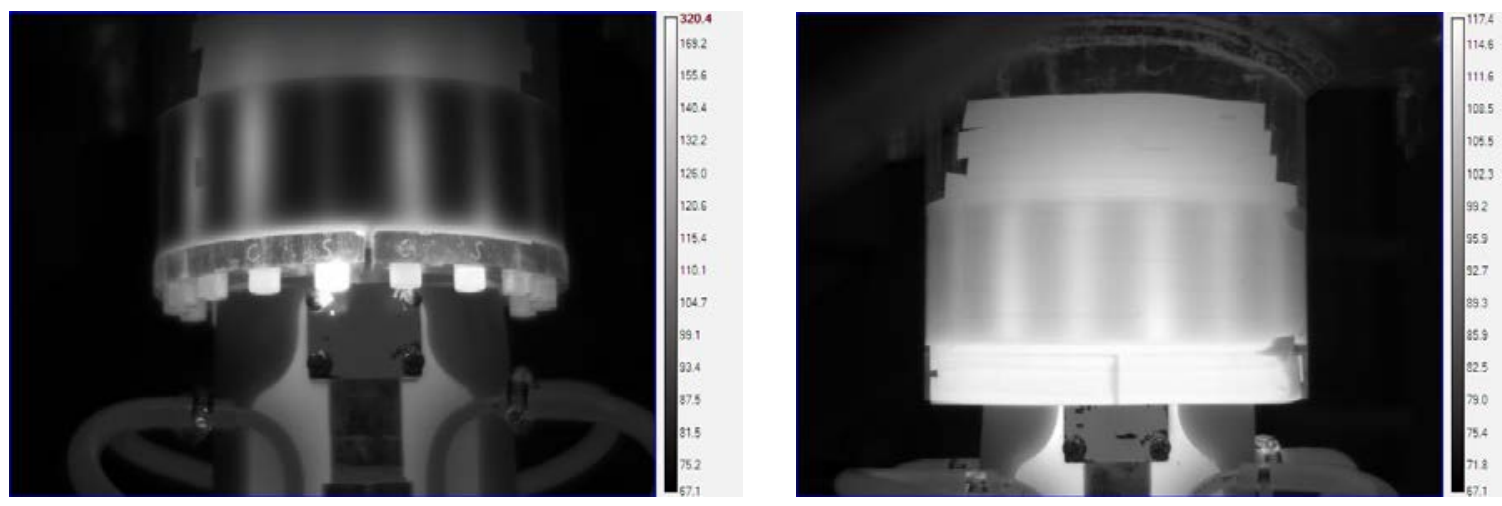
Once the the configuration changes to the feedthru were completed, NTREES was started and power was applied to the dummy grapite test piece. Temperatures in the dummy element approaching $2100 \mathrm{~K}$ were achieved at about $120 \mathrm{~kW}$. Temperatures were held at this level for about 30 minutes before NTREES was shutdown. A view of the the dummy test element can be seen in Figure 6.

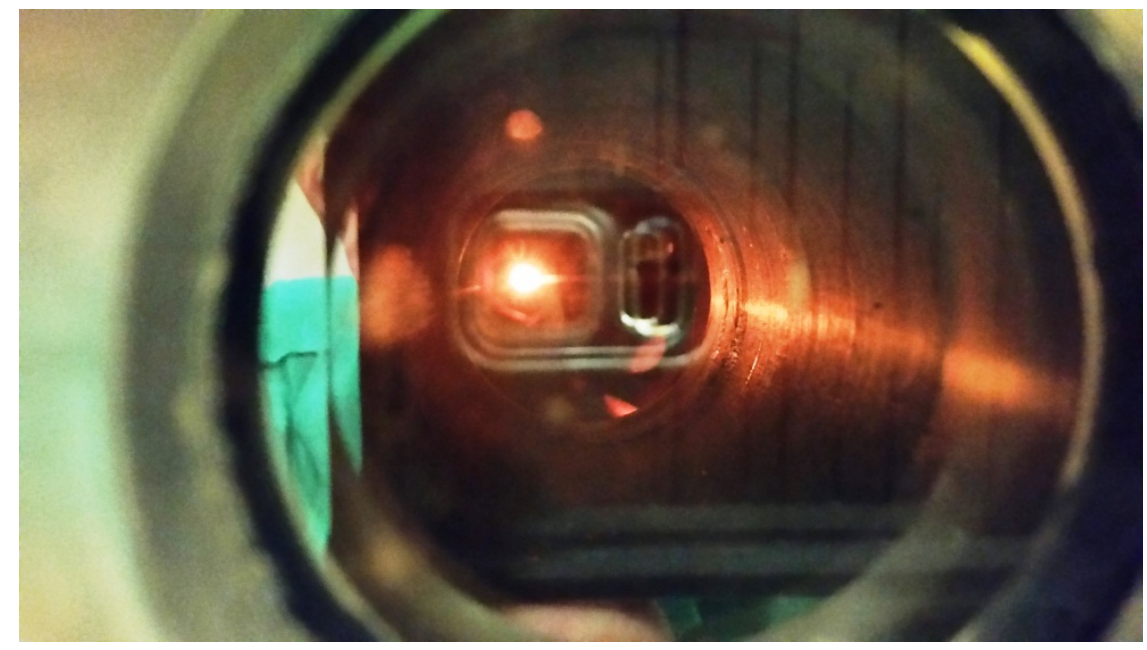

Figure 6 Dummy Element Under Test in NTREES

At the conclusion of the power system checkouts, NTREES was disassembled to mount hot and cold clamping systems in the NTREES chamber to allow more prototypical fuel elements to be mounted in the chamber and subjected to loading forces similar to what they would seen in a reactor system. The clamping system allow a fuel element to be loaded into its holder and positioned appropriately in the coil and locked into the hot receiver portion. Testing is expected to commence in early August 2014.

\section{Conclusion}

The newly upgraded NTREES facility provides a means to realistically test these fuel elements, even to the point of failure for extremely little cost. Such testing will allow the best fuel designs to quickly move forward to more highly realistic testing in nuclear test facilities with high confidence in their ultimate performance capabilities. The new capabilities of the NTREES system allows prototypic fuel elements to be tested under conditions which more closely simulate the actual conditions they would encounter in an operating nuclear rocket engine.

\section{Acknowledgments}

The NTREES facility upgrade and test activities are funded through Science \& Technology Office at the Marshall Space Flight Center under the Nuclear Cryogenic Propulsion Stage program. The author greatly appreciates the support, both technical and financial, given by that office.

\section{References}

${ }^{1}$ Emrich, W. J., "Nuclear Thermal Rocket Element Environmental Simulator (NTREES)," Space Technology and Applications International Forum: $25^{\text {th }}$ Symposium on Space Nuclear Power and Propulsion, Albuquerque, NM, Ed. Mohamed S. El-Genk, 2008, pp. 541-548. 